Hardy-Ramanujan Journal

Vol.24 (2001) 9-17

\title{
On the values of the Riemann zeta-function at rational arguments
}

\author{
S. Kanemitsu, Y. Tanigawa and M. Yoshimoto \\ Dedicated to Professor Takashi Yanagawa birthday with great respect
}

\begin{abstract}
In our previous papers [3], [4] we obtained a closed form evaluation of Ramanujan's type of the values of the (multiple) Hurwitz zeta-function at rational arguments (with denominator even and numerator odd), which was in turn a vast generalization of D. Klusch's and M. Katsurada's generalization of Ramanujan's formula. In this paper we shall continue our pursuit, specializing to the Riemann zeta-function, and obtain a closed form evaluation thereof at all rational arguments, with no restriction to the form of the rationals, in the critical strip. This is a complete generalization of the results of the aforementioned two authors. We shall obtain as a byproduct some curious identities among the Riemann zetavalues.
\end{abstract}

\section{Introduction and notation}

In this paper we shall give a closed form evaluation of Ramanujan's type of the values of the Riemann zeta-function at positive rational arguments in the critical strip.

We have launched on this evaluation problem of zeta-values at rational arguments in [2], where we have succeeded in getting a Ramanujan type formula for $\zeta(2 / 3)$ after examining the results of D. Klusch [6] and M. Katsurada [5].

In [3] we have evaluated the values of the Hurwitz zeta-function at rational arguments smaller than 1 (of the form $1-b / a$, with $b$ odd, $a$ even), which we have transformed into

2000 Mathematics Subject Classification: 11M35,11M06

The first and third authors are supported by Grand-in-Aid for Scientific Research (C) 1164005 and the Sumitomo Foundation fellowship 000406 respectively. 
the evaluation of values of the multiple Hurwitz zeta-function through the Mellin-Vardi decomposition thereof into a linear combination of Hurwitz zeta-functions.

On the other hand, in [4] we have adopted a method closer to Katsurada's to obtain the evaluation of the multiple Hurwitz zeta-f unction at positive rational arguments $b / a$.

In this paper we shall push forward our approach in [2] to the general situation and evaluate the values of the Riemann zeta-function at positive rational arguments, with no restriction to the form, thus supplying new information on them for $\zeta(b / a)$ with $b$ even, $a$ odd or both odd, missing in all preceding papers [2]-[4] (cf. Corollary 1).

We obtain as by-product some curious identities among modified Lambert series which look non-trivial and which follow by equating two expressions for them (cf. Corollary 2).

For readers' convenience of comparison of the results in this paper and those in [2]-[4] we shall use the same notation and give the reference to the corresponding formulas in the latter.

Notation. Let $0 \leq h \leq N$ be integers. We put

$$
\begin{gathered}
a_{j, N}=\cos \left(\frac{\pi j}{2 N}\right), \quad b_{j, N}=\sin \left(\frac{\pi j}{2 N}\right) \\
A_{N}(y)=\pi(2 \pi y)^{\frac{1}{N}} \\
f_{0}(x ; n, N)=\frac{e^{-A_{N}\left(\frac{n}{x}\right)}}{2 \sinh \left(A_{N}\left(\frac{n}{x}\right)\right)}
\end{gathered}
$$

and

$$
f_{j}(x ; n, N, h)=\frac{\cos \left(2 A_{N}\left(\frac{n}{x}\right) b_{j, N}+\frac{\pi(2 h-1) j}{2 N}\right)-e^{-2 A_{N}\left(\frac{n}{x}\right) a_{j, N}} \cos \left(\frac{\pi(2 h-1) j}{2 N}\right)}{\cosh \left(2 A_{N}\left(\frac{n}{x}\right) a_{j, N}\right)-\cos \left(2 A_{N}\left(\frac{n}{x}\right) b_{j, N}\right)}
$$

for $j \geq 1$.

\section{Values of the Riemann zeta-function}

We shall prove the following Ramanujan type formula for $\zeta\left(\frac{N-2 h+1}{N}\right)$ on the lines as close as possible to those of proofs of the previous results. In the proof, we shall give the corresponding formula numbers in [2] and [4] (since those in [4] are suggestive of the corresponding ones in [3]). 
Theorem 1. Let $N$ and $h$ be fixed natural numbers with $h \leq N / 2$. Then we have for $x>0$,

$$
\sum_{n=1}^{\infty} \frac{n^{N-2 h}}{e^{n^{N} x}-1}=P(x)+S(x)
$$

where

$$
\begin{aligned}
P(x)= & P(x ; N, h)=-\frac{1}{2} \zeta(-N+2 h)+\zeta(2 h) x^{-1} \\
& +\frac{1}{N} \Gamma\left(\frac{N-2 h+1}{N}\right) \zeta\left(\frac{N-2 h+1}{N}\right) x^{-\frac{N-2 h+1}{N}}
\end{aligned}
$$

and

$$
\begin{aligned}
S(x) & =S(x ; N, h)=\frac{(-1)^{h+1}}{N}\left(\frac{2 \pi}{x}\right)^{\frac{N-2 h+1}{N}} \\
& \times \sum_{n=0}^{\infty} \frac{1}{n^{\frac{2 h-1}{N}}}\left\{f_{0}(x ; n, N)+\sum_{j=1}^{\frac{N-1}{2}} f_{2 j}(x ; n, N, h)\right\}
\end{aligned}
$$

for $N$ odd, and

$$
\begin{aligned}
S(x) & =S(x ; N, h) \\
& =\frac{(-1)^{h+1}}{N}\left(\frac{2 \pi}{x}\right)^{\frac{N-2 h+1}{N}} \sum_{n=0}^{\infty} \frac{1}{n^{\frac{2 h-1}{N}}} \sum_{j=1}^{\frac{N}{2}} f_{2 j-1}(x ; n, N, h),
\end{aligned}
$$

for $N$ even, with $f_{j}$ defined by (1.3) and (1.4).

Proof. We consider the integral with $c_{0}>1$

$$
I(x)=\frac{1}{2 \pi i} \int_{\left(c_{0}\right)} \Gamma(s) \zeta(s) \zeta(N s-(N-2 h)) x^{-s} d s
$$

where $\left(c_{0}\right)$ denotes the vertical line $\sigma=c_{0},-\infty<t<\infty$ (cf. [2],[4,(2.6)*]).

On the one hand, we have

$$
I(x)=\sum_{n=1}^{\infty} \frac{n^{N-2 h}}{e^{n^{N} x}-1},
$$

i.e. the left-hand side of (2.1) (cf.[2], [4,(2.6)*]). 
We now shift the line of integration to $(-c)$ with $c>0$, whereby we encounter the poles of the integrand at $s=0,1, \frac{N-2 h+1}{N}$, whence we deduce that

$$
I(x)=P(x)+J(x)
$$

where

$$
J(x)=J_{-c}(x)=\frac{1}{2 \pi i} \int_{(-c)} \Gamma(s) \zeta(s) \zeta(N s-(N-2 h)) x^{-s} d s
$$

and $P(x)$ is given by $(2.2)$ (cf.[2], $\left[4,(2.8)_{1}^{*}\right]$ ).

Hence it is enough to prove that $J(x)$ coincides with $S(x)$.

We make the change of variables $s \leftrightarrow 1-s$ and use the functional equation for the Riemann zeta-function in the form

$$
\begin{array}{r}
\Gamma(1-s) \zeta(1-s) \zeta(2 h-N s)=(2 \pi)^{2 h-(N+1) s}(-1)^{h+1} C_{N}\left(\frac{\pi s}{2}\right) \\
\times \Gamma(N s-2 h+1) \zeta(N s-2 h+1) \zeta(s)
\end{array}
$$

(cf. [2], $\left.\left[4,(2.9)^{*}\right]\right)$, where $C_{N}(z)$ denotes the Chebyshev polynomial

$$
C_{N}(z)=\frac{\sin N z}{\sin z}=\sum_{j=-(N-1)}^{N-1} " e^{i j z},
$$

and where $\sum^{\prime \prime}$ means the summation over $j$ which increases by 2 . Hence the integrand of

$$
J(x)=\frac{1}{2 \pi i} \int_{(1+c)} \Gamma(1-s) \zeta(1-s) \zeta(2 h-N s) x^{-(1-s)} d s
$$

becomes

$$
\begin{aligned}
G(s) x^{-(1-s)} & =(-1)^{h+1}(2 \pi)^{2 h-(N+1) s} C_{N}\left(\frac{\pi s}{2}\right) \\
\times & \Gamma(N s-2 h+1) \zeta(N s-2 h+1) \zeta(s) x^{-(1-s)} .
\end{aligned}
$$

Now make the change of variables

$$
N s-2 h+1=s_{1}, \quad s=\frac{s_{1}+2 h-1}{N} .
$$

Then $G(s) x^{s-1}$ becomes

$$
\begin{aligned}
G\left(\frac{s_{1}+2 h-1}{N}\right) x^{-1+\frac{s_{1}+2 h-1}{N}}=(-1)^{h+1}\left(\frac{2 \pi}{x}\right)^{\frac{N-2 h+1}{N}}\left(\frac{x}{(2 \pi)^{N+1}}\right)^{\frac{s_{1}}{N}} \\
\times \Gamma\left(s_{1}\right) \zeta\left(s_{1}\right) \zeta\left(\frac{s_{1}+2 h-1}{N}\right) C_{N}\left(\frac{\pi\left(s_{1}+2 h-1\right)}{2 N}\right) .
\end{aligned}
$$


Incorporating these, we successively get

$$
\begin{aligned}
J(x) & =\frac{1}{2 \pi i} \int_{(1+c)} G(s) x^{s-1} d s \\
= & (-1)^{h+1}\left(\frac{2 \pi}{x}\right)^{\frac{N-2 h+1}{N}} \frac{1}{2 \pi i} \int_{\left(c_{1}\right)}\left(\frac{(2 \pi)^{N+1}}{x}\right)^{-\frac{s_{1}}{N}} \Gamma\left(s_{1}\right) \\
& \times \zeta\left(s_{1}\right) \zeta\left(\frac{s_{1}+2 h-1}{N}\right) C_{N}\left(\frac{\pi\left(s_{1}+2 h-1\right)}{2 N}\right) \frac{d s_{1}}{N}
\end{aligned}
$$

where $c_{1}=N(1+c)-2 h+1$ (cf. $\left.[2,(3.7)],\left[4,(2.18)_{1}^{*},(2.18)_{2}^{*}\right]\right)$.

We substitute the Dirichlet series

$$
\zeta(s) \zeta\left(\frac{s+2 h-1}{N}\right)=\sum_{m, n=1}^{\infty} n^{-\frac{2 h-1}{N}}\left(m n^{\frac{1}{N}}\right)^{-s}
$$

in (2.12) to obtain

$$
J(x)=\frac{(-1)^{h+1}}{N}\left(\frac{2 \pi}{x}\right)^{\frac{N-2 h+1}{N}} \sum_{m, n=1}^{\infty} n^{-\frac{2 h-1}{N}} E\left(X_{m, n}\right)
$$

(cf. [2], $\left.\left[4,(2.18)_{3}^{*},(2.19)^{*}\right]\right)$, where $\left(\kappa=c_{1}\right)$

$$
E\left(X_{m, n}\right)=\frac{1}{2 \pi i} \int_{(\kappa)} X_{m, n}^{-s} C_{N}\left(\frac{\pi(s+2 h-1)}{2 N}\right) \Gamma(s) d s
$$

(cf. $\left.[2,(3.9)]\left[4,(2.21)^{*}\right]\right)$ with $X_{m, n}$ denoting

$$
X_{m, n}=2 \pi m\left(\frac{2 \pi n}{x}\right)^{\frac{1}{N}}=2 m A_{N}\left(\frac{n}{x}\right),
$$

where $A_{N}(n / x)$ is defined by $(1.3)$ (cf. $\left.\left[4,(2.22)^{*}\right]\right)$.

To evaluate $E\left(X_{m, n}\right)$ we use (2.10) to write

$$
X_{m, n}^{-s} C_{N}\left(\frac{\pi(s+2 h-1)}{2 N}\right)=\sum_{j=-(N-1)}^{N-1} " e^{\frac{\pi i(2 h-1) j}{2 N}}\left(X_{m, n} e^{-\frac{\pi i j}{2 N}}\right)^{-s}
$$

with

$$
\left|\arg X_{m, n} e^{-\frac{\pi i j}{2 N}}\right|<\frac{\pi}{2}
$$

Hence the well-known Mellin inversion formula

$$
\frac{1}{2 \pi i} \int_{(\kappa)} \Gamma(s) Y^{-s} d s=e^{-Y}
$$


applies, and we have

$$
E\left(X_{m, n}\right)=\sum_{j=-(N-1)}^{N-1} e^{\frac{\pi i(2 h-1) j}{2 N}}\left(e^{-2 A_{N}\left(\frac{n}{x}\right) e^{-\frac{\pi i j}{2 N}}}\right)^{m}
$$

(cf. $\left.\left[4,(2.24)^{*}\right]\right)$.

Substituting (2.17) in (2.13) and summing the geometric progression in $m$, we deduce that

$$
\begin{aligned}
J(x)= & \frac{(-1)^{h+1}}{N}\left(\frac{2 \pi}{x}\right)^{\frac{N-2 h+1}{N}} \sum_{j=-(N-1)}^{N-1} " e^{\frac{\pi i(2 h-1) j}{2 N}} \\
& \quad \times \sum_{n=1}^{\infty} \frac{1}{n^{\frac{2 h-1}{N}}} \frac{1}{e^{2 A_{N}\left(\frac{n}{x}\right) e^{-\frac{\pi i j}{2 N}}-1}} \\
= & \frac{(-1)^{h+1}}{N}\left(\frac{2 \pi}{x}\right)^{\frac{N-2 h+1}{N}} \sum_{n=1}^{\infty} n^{-\frac{2 h-1}{N}} \sum_{j=-(N-1)}^{N-1} " Z_{j, n}(x),
\end{aligned}
$$

where

$$
Z_{j, n}(x)=\frac{e^{\frac{\pi i(2 h-1) j}{2 N}} e^{-A_{N}\left(\frac{n}{x}\right) e^{-\frac{\pi i j}{2 N}}}}{2 \sinh \left(A_{N}\left(\frac{n}{x}\right) e^{-\frac{\pi i j}{2 N}}\right)}
$$

(cf. $\left.\left[4,(2.30)^{*}\right]\right)$.

We sum $Z_{j}$ 's in pairs : $Z_{j, n}(x)+Z_{-j, n}(x)$. Distinguishing two cases according to the parity of $N$, we deduce that $J(x)$ coincides with $S(x)$ in (2.3) and (2.4) (cf. [4,(2.31)-(2.34)]

Corollary 1. Under the same notation as in Theorem 1, let $L(x ; N, h)$ be the function defined by

$$
L(x ; N, h)=\sum_{n=1}^{\infty} \frac{n^{N-2 h}}{e^{n^{N} x}-1}-S(x ; N, h)+\frac{1}{2} \zeta(-N+2 h)-\zeta(2 h) x^{-1} .
$$

Then we have

$$
\zeta\left(\frac{N-2 h+1}{N}\right)=\frac{N}{\Gamma\left(\frac{N-2 h+1}{N}\right)} x^{\frac{N-2 h+1}{N}} L(x ; N, h)
$$

and

$$
\zeta\left(\frac{2 h-1}{N}\right)=\frac{N}{\pi}(2 \pi)^{\frac{2 h-1}{N}} \sin \frac{\pi(2 h-1)}{2 N} x^{\frac{N-2 h+1}{N}} L(x ; N, h)
$$

Proof. The first assertion is just the restatement of Theorem 1 and the second assertion follows from the functional equation of the Riemann zeta-function. 


\section{$3 \quad$ Identities among Lambert series}

We shall prove the following consequence of our Corollary 1 which may be of interest for its own sake.

Corollary 2. In the notation of Corollary 1, we have

$$
l L\left(x ; l N, h l-\frac{l-1}{2}\right)=L(x ; N, l)
$$

holds for all $l \geq 1$, odd.

Further suppose $N$ even and put

$$
\tilde{L}(x ; N, h)=\left(\frac{2 \sqrt{\pi}}{x}\right)^{\frac{2 h}{N}} \Gamma\left(\frac{2 h-1+N}{2 N}\right) L(x ; N, h) .
$$

Then

$$
\tilde{L}(x ; N, h)=\tilde{L}(x ; N, N / 2-h+1)
$$

holds for all $h, 1 \leq h \leq N / 2$.

Proof. Formula (3.1) follows from two distinct representations of $\zeta(s)$ at

$$
s=1-\frac{2 h-1}{N}=1-\frac{2\left(h l-\frac{l-1}{2}\right)-1}{N l} .
$$

for any odd integer $l$.

We now turn to (3.3) and suppose $N$ even. Equate the expressions for $\zeta\left(\frac{2 h-1}{N}\right)$ given by $(2.20)$ and by $(2.21)$ with $h$ replaced by $h_{1}=\frac{N}{2}-h+1$ to obtain

$$
\frac{N}{\pi}(2 \pi)^{\frac{2 h-1}{N}} \sin \frac{\pi(2 h-1)}{2 N} x^{\frac{N-2 h+1}{N}} L(x ; N, h)=\frac{N}{\Gamma\left(\frac{N-2 h_{1}+1}{N}\right)} x^{\frac{N-2 h_{1}+1}{N}} L\left(x ; N, h_{1}\right)
$$

or

$$
\frac{1}{\pi}(2 \pi)^{\frac{2 h-1}{N}} \sin \frac{\pi(2 h-1)}{2 N} \Gamma\left(\frac{2 h-1}{N}\right) x^{\frac{N-4 h+2}{N}} L(x ; N, h)=L\left(x ; N, h_{1}\right) .
$$

By the duplication formula and the reciprocity relation of the gamma function, we see that

$$
\sin \frac{\pi(2 h-1)}{2 N} \Gamma\left(\frac{2 h-1}{N}\right)=2^{\frac{2 h-1}{N}-1} \sqrt{\pi} \frac{\Gamma\left(\frac{2 h-1+N}{2 N}\right)}{\Gamma\left(\frac{2(N / 2-h+1)-1+N}{2 N}\right)},
$$


and therefore (3.4) becomes

$$
\begin{aligned}
& \frac{1}{\pi}(2 \pi)^{\frac{2 h-1}{N}} 2^{\frac{2 h-1}{N}-1} \sqrt{\pi} x^{\frac{N-4 h+2}{N}} \Gamma\left(\frac{2 h-1+N}{2 N}\right) L(x ; N, h) \\
& =\Gamma\left(\frac{2(N / 2-h+1)-1+N}{2 N}\right) L(x ; N, N / 2-h+1) .
\end{aligned}
$$

Combing (3.2) and (3.5) implies the assertion (3.3). This completes the proof.

Remark 1.It may be worth recording that the main deformation in (3.1) occurs in the first summation and the coefficients $f_{j}$ 's in the definition of $L$, i.e. in place of $\sum_{n=1}^{\infty} \frac{n^{N-2 h}}{e^{n^{N} x}-1}$ and $f_{j}$ 's, we have

$$
\sum_{n=1}^{\infty} \frac{n^{(N-h+1) l-1}}{e^{n^{N l} x}-1},
$$

and

$$
\begin{aligned}
& f_{j}\left(x ; n, l N, h l-\frac{l-1}{2}\right) \\
& =\frac{\cos \left(2 A_{l N}\left(\frac{n}{x}\right) \sin \frac{\pi j}{2 l N}+\frac{\pi(2 h-1) j}{2 N}\right)-e^{-2 A_{l N}\left(\frac{n}{x}\right) \cos \frac{\pi j}{2 l N} \cos \left(\frac{\pi(2 h-1) j}{2 N}\right)}}{\cosh \left(2 A_{l N}\left(\frac{n}{x}\right) \cos \frac{\pi j}{2 l N}\right)-\cos \left(2 A_{l N}\left(\frac{n}{x}\right) \sin \frac{\pi j}{2 l N}\right)}
\end{aligned}
$$

respectively.

Examples (i) In (3.1), we take $N=2, h=1, l=3$. Then we get $L(x ; 2,1)=3 L(x ; 6,2)$. More explicitly, it reads that

$$
\begin{aligned}
& \sum_{n=1}^{\infty} \frac{1}{e^{n^{2} x}-1}-\frac{1}{2}\left(\frac{2 \pi}{x}\right)^{\frac{1}{2}} \sum_{n=1}^{\infty} \frac{f_{1}(x ; n, 2,1)}{\sqrt{n}}-\frac{1}{4}-\frac{\pi^{2}}{6 x} \\
& =3 \sum_{n=1}^{\infty} \frac{n^{2}}{e^{n^{6} x}-1}+\frac{1}{2}\left(\frac{2 \pi}{x}\right)^{\frac{1}{2}} \sum_{n=1}^{\infty} \frac{1}{\sqrt{n}}\left\{f_{1}(x ; n, 6,2)+f_{3}(x ; n, 6,2)\right. \\
& \left.\quad+f_{5}(x ; n, 6,2)\right\}-\frac{\pi^{4}}{30 x} .
\end{aligned}
$$

(ii) Next we take $N=4, h=1$ in $(3.2)$. Then $\tilde{L}(x ; 4,1)=\tilde{L}(x ; 4,2)$. This means that

$$
\begin{aligned}
\Gamma\left(\frac{5}{8}\right)\left\{\sum_{n=1}^{\infty} \frac{n^{2}}{e^{n^{4} x}-1}-\frac{1}{4}\left(\frac{2 \pi}{x}\right)^{\frac{3}{4}} \sum_{n=1}^{\infty} \frac{1}{n^{1 / 4}}\left(f_{1}(x ; n, 4,1)+f_{3}(x ; n, 4,1)\right)-\frac{\pi^{2}}{6 x}\right\} \\
=\left(\frac{2 \sqrt{\pi}}{x}\right)^{\frac{1}{2}} \Gamma\left(\frac{7}{8}\right)\left\{\sum_{n=1}^{\infty} \frac{1}{e^{n^{4} x}-1}-\frac{1}{4}-\frac{\pi^{4}}{90 x}+\frac{1}{4}\left(\frac{2 \pi}{x}\right)^{\frac{1}{4}}\right. \\
\left.\times \sum_{n=1}^{\infty} \frac{1}{n^{3 / 4}}\left(f_{1}(x ; n, 4,2)+f_{3}(x ; n, 4,2)\right)\right\} .
\end{aligned}
$$




\section{References}

[1] S. Kanemitsu, Y. Tanigawa and M. Yoshimoto, On rapidly convergent series for the Riemann zeta-values via the modular relation, preprint.

[2] S. Kanemitsu, Y. Tanigawa and M. Yoshimoto, On zeta- and $L$-function values at special rational arguments via the modular relation, to appear.

[3] S. Kanemitsu, Y. Tanigawa and M. Yoshimoto, On multiple Hurwitz zeta-function values at rational arguments I, preprint.

[4] S. Kanemitsu, Y. Tanigawa and M. Yoshimoto, On multiple Hurwitz zeta-function values at rational arguments II, preprint.

[5] M. Katsurada, On an asymptotic formula of Ramanujan for a certain theta-type series, Acta Arith. 97 (2001), 157-172.

[6] D. Klusch, On Entry 8 of Chapter 15 of Ramanujan's Notebook II, Acta Arith. 58 (1991), 59-64.

Shigeru Kanemitsu

Graduate School of Advanced Technology

University of Kinki, Iizuka

Fukuoka, 820-8555, Japan

e-mail: kanemitu@fuk.kindai.ac.jp

Yoshio Tanigawa

Graduate School of Mathematics

Nagoya University

Nagoya, 464-8602, Japan

e-mail: tanigawa@math.nagoya-u.ac.jp

Masami Yoshimoto

Research Institute for Mathematical Sciences

Kyoto University

Kyoto, 606-8502, Japan

e-mail: yosimoto@kurims.kyoto-u.ac.jp 